\title{
Capacidad del ArgenSCORE para predecir mortalidad en pacientes mexicanos sometidos a cirugía cardiovascular abierta
}

\section{Capacity of the ArgenSCORE to predict mortality in Mexican patients undergoing open cardiovascular surgery}

\author{
Alicia A. Trujillo-Malo ${ }^{1 *}$, Víctor M. Gudiño-Carballo y Miriam Navarrete-Sandoval ${ }^{2}$ \\ ${ }^{1}$ Facultad Mexicana de Medicina, Universidad La Salle; ${ }^{2}$ Servicio de Terapia Intensiva, Hospital Regional "Gral. Ignacio Zaragoza", Instituto de \\ Seguridad y Servicios Sociales de los Trabajadores del Estado (ISSSTE). Ciudad de México, México
}

\begin{abstract}
Resumen
Introducción: Las escalas EuroSCORE II (European System for Cardiac Operation Risk Evaluation II), STS (de la Society of Thoracic Surgeons) y ArgenSCORE (Argentinean System for Cardiac Operative Risk Evaluation) permiten estimar la mortalidad asociada a cirugía cardiovascular, pero su desempeño es distinto entre poblaciones o estudios. Objetivo: Comparar la capacidad de EuroSCORE II, STS y ArgenSCORE para predecir mortalidad en pacientes mexicanos sometidos a cirugía cardiovascular abierta. Metodología: Se realizó un estudio observacional de cohorte prospectivo en pacientes que fueron sometidos a cirugía cardiovascular en el Hospital General Ignacio Zaragoza ingresados a la unidad de cuidados intensivos (UCI) para cuidados postoperatorios. Se registró información clínica y los puntajes EuroSCORE II, STS y ArgenSCORE al ingreso a UCI. Se registró la mortalidad entre los pacientes y se estimó la capacidad de EuroSCORE II, STS y ArgenSCO$R E$ para predecir mortalidad mediante curvas ROC y regresión logística binaria. Resultados: Se incluyeron 74 pacientes de edad media $58.5 \pm 12.5$ años (41.3\% de sexo femenino); el 57.3\% fallecieron. La mortalidad estimada por EuroSCORE II, STS y ArgenSCORE fue $2.8 \pm 5.6 \%, 3.2 \pm 15.8 \%$ y $6.3 \pm 10.2 \%$, respectivamente. El área bajo la curva (AUC) para EuroSCORE II fue 0.530, para STS 0.508, y para ArgenSCORE 0.550. Por análisis multivariante, el único factor independientemente asociado a mortalidad fue el STS con un $\operatorname{Exp}(B)=4.1$ (IC 95\%: 1.13-14.76; $p=0.032$ ). Con un modelo final que incluyó el sexo, la prioridad quirúrgica y los tres puntajes se mejoró la estimación de predicción de la mortalidad de forma signiffcativa para una AUC final de 0.710. Conclusiones: Todos los modelos evaluados infraestimaron la mortalidad real y se desempeñaron pobremente para predecir mortalidad en pacientes sometidos a cirugía cardiovascular abierta. Se sugiere calibrar y validar los modelos predictivos a distintos escenarios clínicos.
\end{abstract}

Palabras clave: Cirugía cardiovascular. Mortalidad, predicción. EuroSCORE. STS. ArgenSCORE.

\begin{abstract}
Introduction: The EuroSCORE II, STS and ArgenSCORE scales allow estimating mortality related to cardiovascular surgery, but their performance differs between populations or studies. Objective: To compare the capacity of the EuroSCORE II, STS and ArgenSCORE to predict mortality in Mexican patients undergoing open cardiovascular surgery. Methodology: An observational prospective cohort study was carried out in patients who underwent cardiovascular surgery at the Hospital "Gral.
\end{abstract}

Correspondencia:

*Alicia A.Trujillo Malo

E-mail: alexmalo_9@msn.com
Disponible en internet: 17-12-2021 Rev Hosp Jua Mex. 2021;88(4):187-192

www.revistahospitaljuarez.com 1405-9622/@ 2021 Sociedad Médico-Quirúrgica del Hospital Juárez de México, A.C. Publicado por Permanyer. Este es un artículo open access bajo la licencia CC BY-NC-ND (http://creativecommons.org/licenses/by-nc-nd/4.0/). 
Ignacio Zaragoza" were admitted to the ICU for post-operative care. At ICU admission, clinical information and EuroSCORE, STS and ArgenSCORE punctuation were registered. Mortality among patients was recorded and the ability of the EuroSCORE II, STS and ArgenSCORE punctuation to predict mortality was estimated using ROC curves and binary logistic regression. Results: 74 patients of mean age $74.4 \pm 10.5$ years (41.3\% female and male) were included; $58.1 \%$ of the patients died. Mortality estimated by EuroSCORE II, STS and ArgenSCORE was $2.8 \pm 5.6 \%, 3.2 \pm 15.8 \%$ and $6.3 \pm 10.2 \%$, respectively. The AUC for the EuroSCORE II was 0.530, for the STS 0.508, and for the ArgenSCORE 0.550. By multivariate analysis, the only factor independently associated with mortality was STS score with $\operatorname{Exp}(B)=4.1(95 \% \mathrm{Cl} 1.13-14.76, p=0.032)$. A final model that included gender, surgical priority, the 3 punctuations improved the prediction of mortality $(A U C=0.710)$. Conclusions: All the models evaluated underestimated actual mortality and performed poorly in predicting mortality in patients undergoing open cardiovascular surgery. It is suggested to calibrate and validate the predictive models to different clinical scenarios.

Key words: Cardiovascular surgery. Mortality. Prediction. EuroSCORE. STS. ArgenSCORE.

\section{Introducción}

Los grandes avances en cirugía cardiovascular han mejorado la expectativa de vida de los pacientes sometidos a procedimientos como reemplazos valvulares, revascularización coronaria y cierres de comunicaciones entre cavidades cardiacas ${ }^{1,2}$. Sin embargo, estos procedimientos no están exentos de complicaciones, incluyendo muerte, que es más frecuente en la primer semana postoperatoria ${ }^{3,4}$.

El desarrollo de modelos clínicos predictivos en unidades de cuidados intensivos ( $\mathrm{UCl}$ ) ha representado un avance significativo para los médicos, investigadores clínicos, directores de $\mathrm{UCl}$ y las áreas de calidad hospitalaria ${ }^{5}$.

En cirugía cardiovascular se han propuesto diversos modelos para predecir la mortalidad posterior a cirugía cardiovascular, incluyendo los modelos EuroSCORE II (European System for Cardiac Operation Risk Evaluation II), STS (Society of Thoracic Surgeons) y ArgenSCORE (Argentinean System for Cardiac Operative Risk Evaluation) $)^{6,7}$. Si bien estos modelos han sido validados externamente, su desempeño no es el esperado en tales validaciones y se han reportado áreas bajo la curva (AUC) de 0.628-0.800 $7-9$. Por lo tanto, es fundamental evaluar si los modelos existentes son buenos predictores y se ajustan a la realidad de cada hospital, ya que en algunos casos los modelos de predicción no han sido tan buenos como se espera $^{10,11}$ y porque se ha demostrado que la calibración de los modelos de predicción de mortalidad cardiovascular se ha deteriorado con el tiempo ${ }^{12}$.

En hospitales mexicanos poco se ha evaluado el desempeño de escalas de predicción de mortalidad postoperatoria en pacientes sometidos a cirugía cardiaca $^{13}$, por lo tanto, el objetivo del presente estudio es comparar la capacidad de EuroSCORE II, STS y
ArgenSCORE para predecir mortalidad en una muestra de pacientes mexicanos sometidos a cirugía cardiovascular.

\section{Método}

El presente estudio fue sometido a aprobación por el Comité de Investigación y Bioética del Hospital General Ignacio Zaragoza, del Instituto de Seguridad y Servicios Sociales de los Trabajadores del Estado (ISSSTE); el representante legal o familiar responsable de los pacientes firmaron carta de consentimiento informado para incluir al paciente en el estudio.

Se realizó un estudio observacional de cohorte, prospectivo, en el que se incluyeron pacientes sometidos a cirugía cardiovascular abierta en el Hospital General Ignacio Zaragoza que fueron ingresados a la Unidad de Cuidados Intensivos en el periodo enero de 2017 - diciembre de 2018. Se excluyeron pacientes que fueron sometidos a cirugía mínimamente invasiva y se eliminaron aquellos con información incompleta al final del estudio.

Se registró la siguiente información de interés al ingreso a la UCl: edad, sexo, peso, índice de masa corporal (IMC), comorbilidades, fracción de eyección, antecedente de patología cardiovascular, procedimiento quirúrgico realizado, complicaciones transoperatorias y los puntajes EuroSCORE II, STS y ArgenSCORE. Se monitorizó a los pacientes hasta su egreso, registrando la mortalidad.

El análisis estadístico se realizó en SPSS v.25 para Mac, mediante pruebas descriptivas e inferenciales. El análisis descriptivo se realizó con media y desviación estándar para variables cuantitativas, y con frecuencias y porcentajes para variables cualitativas. Como pruebas estadísticas inferenciales se utilizaron: $t$ de Student y chi cuadrada. Se calculó el AUC de los puntajes 
Tabla 1. Características clínicas generales de los pacientes

\begin{tabular}{|c|c|}
\hline Característica & Valores \\
\hline Edad (años) & $58.5 \pm 12.5$ \\
Sexo, \% (n) & $41.3(31)$ \\
Femenino & $58.7(44)$ \\
Masculino & $74.4 \pm 10.5$ \\
Peso (kg) & $27.9 \pm 2.9$ \\
IMC (kg/m²) & \\
Comorbilidades & $37.3(28)$ \\
Hipertensión arterial sistémica & $41.3(31)$ \\
Diabetes mellitus & $4.0(3)$ \\
Enfermedad pulmonar & $2.7(2)$ \\
Enfermedad renal crónica & \\
Antecedente de enfermedades & $33.3(25)$ \\
cardiovasculares & $41.3(31)$ \\
Enfermedad arterial coronaria & $24.0(18)$ \\
Infarto al miocardio & $22.7(17)$ \\
Alteraciones del ritmo cardiaco & $2.7(2)$ \\
Hipertensión arterial pulmonar & \\
Cirugía cardiaca previa & $1.4 \pm 2.1$ \\
Características preoperatorias & $79.2 \pm 26.5$ \\
Creatinina sérica (mg/dl) & \\
Aclaramiento de creatinina (ml/min) & $51.7 \pm 12.4$ \\
FEVI (\%) & $8.3(6)$ \\
Clasificación NYHA, \%(n) & $47.2(34)$ \\
I & $44.4(32)$ \\
II & $0.0(0)$ \\
III & \\
IV & \\
\hline IV &
\end{tabular}

IMC: índice de masa corporal; FEVI: fracción de eyección del ventrículo izquierdo.

EuroSCORE II, STS y ArgenSCORE para predecir mortalidad. Mediante regresión logística binaria con prueba de Hosmer-Lemeshow se identificaron factores independientemente asociados a mortalidad y se creó un modelo final de predicción. Una $p<0.05$ se consideró significativa.

\section{Resultados}

Se incluyeron un total de 74 pacientes que fueron sometidos a cirugía cardiovascular de edad promedio $58.5 \pm 12.5$ años. El $41.3 \%$ de los pacientes eran femeninos y el $58.7 \%$ masculinos. El peso e IMC promedio, las comorbilidades y el antecedente de enfermedades cardiovasculares se presentan en la tabla 1. La fracción de eyección del ventrículo izquierdo (FEVI) promedio fue $51.7 \pm 12.4 \%$, mas de $90 \%$ tuvieron una clasificación NYHA II o III (Tabla 2).

Los procedimientos quirúrgicos realizados a los pacientes fueron sustitución valvular mitral (24\%), sustitución valvular aórtica (36\%), revascularización coronaria $(32 \%)$, cierre de comunicación interauricular $(6.7 \%)$ y resección tumoral de aurícula izquierda (1.3\%).

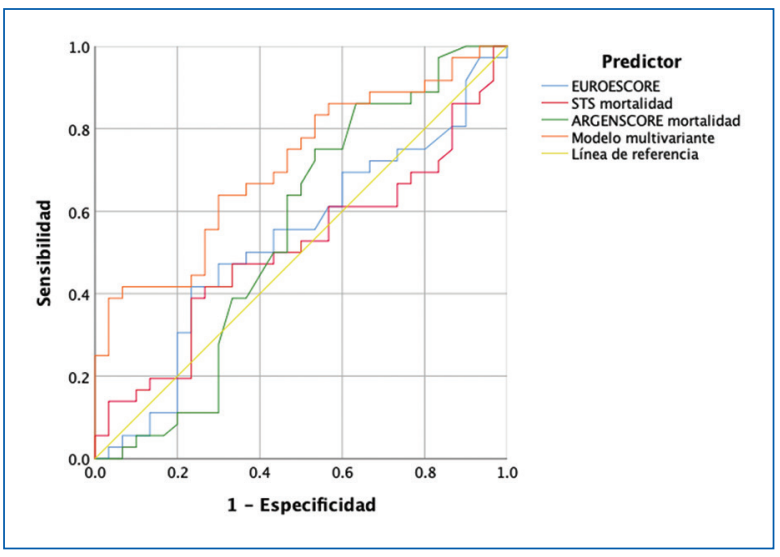

Figura 1. Curvas ROC sobre la capacidad del EuroSCORE II (European System for Cardiac Operation Risk Evaluation II), el índice de la STS (Society of Thoracic Surgeons) y el ArgenSCORE (Argentinean System for Cardiac Operative Risk Evaluation), de forma individual y utilizando un modelo multivariante a partir de las características incluidas en la regresión.

El $71.2 \%$ de las cirugías fueron electivas, el $4.1 \%$ de emergencia y el $24.7 \%$ de urgencia.

La mortalidad fue del $57.3 \%$ de los pacientes $(n=43)$, siendo la mortalidad del $52.3 \%$ entre mujeres y del $61.4 \%$ en hombres $(p=0.492)$. No se encontraron diferencias significativas en la tasa de mortalidad por tipo de procedimiento quirúrgico realizado ( $p=0.891)$. Tampoco se encontraron diferencias significativas en la tasa de mortalidad por grupos de edad ni por categorías de IMC.

La mortalidad promedio estimada con las escalas EuroSCORE II, STS y ArgenSCORE fue $2.8 \pm 5.6,3.2$ \pm 15.8 y $6.3 \pm 10.2$, respectivamente. No hubo diferencias significativas en los puntajes medios entre los que fallecieron y los sobrevivientes (Tabla 2). La capacidad de predicción para mortalidad del EuroSCORE II, STS y ArgenSCORE fue estimada mediante curvas ROC: $0.530,0.508$ y 0.550 , respectivamente (Fig. 1).

Al realizar regresión logística binaria, el único factor significativa e independientemente asociado a mortalidad fue STS con un $\operatorname{Exp}(B)=4.1$ (intervalo de confianza del 95\%: 1.13-14.76; $p=0.032$ ) (Tabla 3). Con este modelo (que incluyó la prioridad quirúrgica, STS, ArgenSCORE y EuroSCORE II) la predicción mejoró, con una AUC de 0.710 (Fig. 1).

\section{Discusión}

En el presente estudio se comparó la capacidad de EuroSCORE II, STS y ArgenSCORE para predecir 
Tabla 2. Comparación de la mortalidad estimada con EuroSCORE II (European System for Cardiac Operation Risk Evaluation II), STS (Society of Thoracic Surgeons) y ArgenSCORE (Argentinean System for Cardiac Operative Risk Evaluation) entre pacientes muertos y sobrevivientes

\begin{tabular}{|l|c|c|c|c|}
\hline & Muertos & Sobrevivientes & Total & Valor de p* \\
\hline EuroSCORE II & $2.3 \pm 2.9$ & $3.5 \pm 7.9$ & $2.8 \pm 5.6$ & 0.391 \\
\hline STS & $4.9 \pm 21.3$ & $1.1 \pm 0.8$ & $3.2 \pm 15.8$ & 0.340 \\
\hline ArgenSCORE & $5.1 \pm 7.8$ & $7.8 \pm 12.6$ & $6.3 \pm 10.2$ & 0.278 \\
\hline
\end{tabular}

*Prueba t de Student.

Tabla 3. Análisis multivariante para identificar factores independientemente asociados a mortalidad

\begin{tabular}{|l|c|c|c|c|}
\hline Factor & -B & ORa & IC 95\% & Valor de $\mathbf{p}^{*}$ \\
\hline STS & 1.405 & 4.077 & $1.13-14.76$ & 0.032 \\
\hline Sexo masculino & -1.350 & 0.259 & $0.063-1.064$ & 0.061 \\
\hline EuroSCORE II & -0.126 & 0.881 & $0.685-1.135$ & 0.327 \\
\hline ArgenSCORE & -0.069 & 0.934 & $0.854-1.020$ & 0.129 \\
\hline $\begin{array}{l}\text { Prioridad } \\
\text { quirúrgica } \\
\text { (emergencia) }\end{array}$ & 0.411 & 1.508 & $0.746-3.049$ & 0.253 \\
\hline Constante & -0.858 & 0.424 & - & - \\
\hline
\end{tabular}

*Prueba t de Student

B: Coeficiente de regresión B; ORa: Odds ratio ajustado; IC 95\%: intervalo de confianza del 95\%; STS: Society of Thoracic Surgeons; EuroSCORE II (European System for Cardiac Operation Risk Evaluation II); ArgenSCORE: Argentinean System for Cardiac Operative Risk Evaluation.

mortalidad en pacientes sometidos a cirugía cardiovascular, encontrando algunos resultados interesantes que se analizan a continuación.

La mortalidad real $(57.3 \%)$ de los pacientes fue muy superior a la estimada con los sistemas EuroSCORE II, STS y ArgenSCORE, y a la reportada en la literatura, si bien la mortalidad reportada en estudios internacionales no supera el $11.5 \%$ y en México no supera el $10 \%^{11,14-16}$, en este estudio la mortalidad fue muy elevada. Si bien se desconoce con precisión la razón de esta elevada mortalidad y podría ser motivo de otra investigación, en el Hospital sede del estudio durante el periodo del estudio se superó una curva de aprendizaje quirúrgico y posiblemente hubo otros factores intrínsecos del hospital que contribuyeron a la elevada mortalidad y a que los modelos EuroSCORE II, STS y ArgenSCORE no se desempeñaran bien para la predicción de mortalidad poscirugía cardiovascular, ya que estos modelos no toman en cuenta factores propios del centro hospitalario donde se emplean.
Ahora bien, dado que la mortalidad es mayor en cirugías de reemplazo valvular que en revascularización $n^{17,18}$ y el $80 \%$ de los procedimientos fueron valvulares, esto pudo contribuir al exceso de mortalidad observado.

El desempeño de los modelos EuroSCORE II, STS y ArgenSCORE fue pobre y muy cercano a 0.5 , lo cual si bien no se esperaba ${ }^{19-21}$ no es raro, dado que en algunos estudios realizados para evaluar la capacidad de predicción de tales modelos el desempeño también ha sido pobre. Holiski et al., por ejemplo, encontraron una AUC de 0.86 para EuroSCORE II y de 0.64 para STS en pacientes alemanes ${ }^{11}$. Kofler et al. encontraron una AUC de 0.664 y 0.628 de los puntajes STS y EuroSCORE II para la predicción de mortalidad ${ }^{10}$. De manera similar, Borde et al. encontraron que tanto el EuroSCORE II como el STS predijeron pobremente mortalidad (AUC: 0.69 de EusoSCORE y 0.65 de STS ${ }^{22}$. Otros estudios realizados en Latinoamérica también han encontrado baja capacidad predictiva del EuroSCORE II y STS ${ }^{6}$. Esto indica que la baja capacidad de predicción del STS y EuroSCORE II encontrada en nuestro estudio es similar a lo encontrado en estos estudios previos. En contraste, nuestro estudio no concuerda con los pocos reportes sobre la capacidad de predicción del ArgenSCORE en estudios de validación externa (AUC: 0.82 y 0.84$)^{7,23}$. Pero nuestros hallazgos sugieren que no se ajusta adecuadamente el modelo ArgenSCORE en nuestro contexto.

Lo anterior refuerza el concepto de que estas escalas no son necesariamente generalizables ni exactas en todos los estudios y que no siempre se replican con la misma capacidad predictora en series de pacientes que son aparentemente de características similares. De hecho, existen limitaciones de los modelos de predicción que se deben principalmente a aspectos estadísticos intrínsecos y de diseño metodológico, a que con frecuencia no toman en cuenta el efecto de la intervención, a que la enfermedad en la $\mathrm{UCl}$ es un 
proceso dinámico y a que en el caso de pacientes postoperados los modelos no toman en cuenta factores propios de la cirugía y la pericia del cirujano ${ }^{5,12,24}$. Por lo tanto, es importante tomar con precaución las estimaciones de mortalidad de los modelos actuales y considerar en futuros modelos de predicción ajustes, calibraciones y validaciones en escenarios clínicos distintos.

En este estudio el único factor asociado significativamente en el análisis multivariante con mortalidad fue el modelo STS, lo cual no es raro, dado que algunos estudios de validación externa de esta herramienta han encontrado que es el modelo con mejor capacidad de predicción $^{7-9,25}$. Finalmente, el análisis multivariante además de ayudarnos a identificar qué factor se asoció independientemente a mortalidad, permite ver que la capacidad de predicción de los modelos puede mejorarse si se consideran como parte de modelos multivariantes que consideren otras características clínicas 0 paraclínicas.

Si bien el presente estudio tiene algunas limitaciones, como es su modesto tamaño de muestra, sí permite evaluar el desempeño pronóstico de los modelos EuroSCORE II, STS y ArgenSCORE en un escenario hospitalario real, de alta mortalidad, y resalta de importancia de calibrar y ajustar los modelos de predicción a cada realidad hospitalaria.

\section{Conclusiones}

Los modelos EuroSCORE II, STS y ArgenSCORE se desempeñaron pobremente para predecir mortalidad entre los pacientes sometidos a cirugía cardiovascular abierta. Todos infraestimaron la mortalidad real, posiblemente porque no tienen en cuenta los factores específicos de cada hospital y/o población. Por lo tanto, es necesario calibrar y validar los modelos a distintos escenarios clínicos.

\section{Agradecimientos}

Al personal de salud que labora en el Servicio de Medicina Crítica, médicos, enfermeras y personal administrativo del Hospital Regional General Ignacio Zaragoza, del ISSSTE, por su incansable labor al cuidado de los pacientes.

\section{Financiamiento}

Los autores declaran no contar con fuentes de financiamiento.

\section{Conflicto de intereses}

Los autores declaran que no existe conflicto de intereses.

\section{Responsabilidades éticas}

Protección de personas y animales. Los autores declaran que para esta investigación no se han realizado experimentos en seres humanos ni en animales.

Confidencialidad de los datos. Los autores declaran que han seguido los protocolos de su centro de trabajo sobre la publicación de datos de pacientes.

Derecho a la privacidad y consentimiento informado. Los autores han obtenido el consentimiento informado de los pacientes y/o sujetos referidos en el artículo. Este documento obra en poder del autor de correspondencia.

\section{Bibliografía}

1. Langer NB, Argenziano M. Minimally invasive cardiovascular surgery: Incisions and approaches. Methodist Debakey Cardiovasc J. 2016; $12(1): 4-9$.

2. Herreros J, Rábago G, Martín Trenor A. Progresos en cirugía cardiaca Rev Med Univ Navarra. 2005;49(3):58-61.

3. Mazzeffi M, Zivot J, Buchman T, Halkos M. In-hospital mortality after cardiac surgery: patient characteristics, timing, and association with postoperative length of intensive care unit and hospital stay. Ann Thorac Surg. 2014;97(4):1220-5

4. Manlhiot C, Rao V, Rubin B, Lee DS. Comparison of cardiac surgery mortality reports using administrative and clinical data sources: a prospective cohort study. CMAJ Open. 2018;6(3):E316-E321.

5. Thibault G. Prognosis and clinical predictive models for critically ill patients. En: Institute of Medicine (US) Committee on Care at the End of Life; Field MJ, Cassel CK E, editores. Approaching death: Improving care at the end of life. Washington, DC: National Academies Press; 1997.

6. Martínez Ochoa CM, Mabel Cañas E, Castro Pérez JA, Saldarriaga Giraldo Cl, González Berrío C, González Jaramillo N. Valor predictivo del EuroSCORE II y el STS score en pacientes sometidos a cirugía cardiaca valvular por el abordaje mínimamente invasivo. Rev Colomb Cardiol. 2016;23:427-34.

7. Carosella VC, Grancelli H, Rodríguez W, Sellanes M, Cáceres M, Cohen Arazi $\mathrm{H}$, et al. Primer puntaje de riesgo latinoamericano en cirugía cardíaca (ArgenSCORE): Validación externa y temporal a 10 años de su desarrollo. Rev Argent Cardiol. 2011;79:500-7.

8. Atashi A, Amini S, Tashnizi MA, Moeinipour AA, Aazami MH, Tohidnezhad F, et al. External validation of European System for Cardiac Operative Risk Evaluation II (EuroSCORE II) for risk prioritization in an Iranian Population. Brazilian J Cardiovasc Surg. 2018; $33(1): 40-6$.

9. Hickey GL, Blackstone EH. External model validation of binary clinical risk prediction models in cardiovascular and thoracic surgery. J Thorac Cardiovasc Surg. 2016;152(2):351-5.

10. Kofler M, Reinstadler SJ, Stastny L, Dumfarth J, Reindl M, Wachter K, et al. EuroSCORE II and the STS score are more accurate in transapical than in transfemoral transcatheter aortic valve implantation. Interact Cardiovasc Thorac Surg. 2018;26(3):413-9.

11. Holinski S, Jessen S, Neumann K, Konertz W. Predictive power and implication of EuroSCORE, EuroSCORE II and STS Score for isolated repeated aortic valve replacement. Ann Thorac Cardiovasc Surg. 2015;21(3):242-6.

12. Hickey GL, Grant SW, Caiado C, Kendall S, Dunning J, Poullis M, et al. Dynamic prediction modeling approaches for cardiac surgery. Circ Cardiovasc Qual Outcomes. 2013;6(6):649-58.

13. Careaga-Reyna G, Martínez-Carballo G, Anza-Costabile LM, Ávila-Funés A Euroscore para predecir morbimortalidad en cirugía cardiaca valvular. Cir Cir. 2008;76:497-505.

14. Beckmann A, Funkat A-K, Lewandowski J, Frie M, Markewitz A Harringer W. German Heart Surgery Report 2016: The Annual Updated Registry of the German Society for Thoracic and Cardiovascular Surgery. Thorac Cardiovasc Surg. 2017;65(07):505-18. 
Rev Hosp Jua Mex. 2021;88(4)

15. Barceló IA, Mestres C. Cirugía cardiovascular en España en los años 2009-2010. Registro de intervenciones de la Sociedad Española de Cirugía Torácica-Cardiovascular (SECTCV). Cir Cardiovasc. 2012;19(3):315-28.

16. Rodríguez-Hernández A, García-Torres $M$, Bucio Reta $E$ Baranda-Tovar FM. Análisis de mortalidad y estancia hospitalaria en cirugía cardiaca en México 2015: datos del Instituto Nacional de Cardiología. Arch Cardiol Mex. 2018;88(5):397-402.

17. Investigadores ESMUCICA. Estudio Multicéntrico de Cirugía Cardiaca. Pacientes valvulares. Rev Argent Cardiol. 2001;69(1):68-79.

18. Investigadores ESMUCICA. Estudio multicéntrico de cirugía cardíaca. Pacientes coronarios. Rev Argent Cardiol. 1999;67(5):606-16.

19. Ad N, Holmes SD, Patel J, Pritchard G, Shuman DJ, Halpin L. Comparison of EuroSCORE II, Original EuroSCORE, and The Society of Thoracic Surgeons Risk Score in Cardiac Surgery Patients. Ann Thorac Surg. 2016;102(2):573-9.

20. Carosella V, Mastantuono C, Golovonevsky V, Cohen V, Grancelli H, Rodríguez W, et al. Validación prospectiva y multicéntrica del ArgenSCORE en la cirugía de reemplazo valvular aórtico. Comparación con e EuroSCORE I y el EuroSCORE II. Rev Argent Cardiol. 2013;82(1):6-12.
21. Carosella VC, Mastantuono C, Golovonevsky V, Cohen V, Grancelli H, Rodríguez W, et al. Validación prospectiva y multicéntrica del ArgenSCORE en la cirugía de reemplazo valvular aórtico. Comparación con el EuroSCORE I y el EuroSCORE II. Rev Arg Cardiol. 2014;82(1):6-12.

22. Borde D, Gandhe U, Hargave N, Pandey K, Khullar V. The application of European system for cardiac operative risk evaluation II (EuroSCORE II) and Society of Thoracic Surgeons (STS) risk-score for risk stratification in Indian patients undergoing cardiac surgery. Ann Card Anaesth. $2013 ; 16(3): 163$

23. Mamberto A, Cipolleti N, Volpi F, et al. Comparación del ArgenSCORE, EuroSCORE II y STS score en la predicción de mortalidad intrahospitalaria en cirugía cardíaca. Revi Cons Arg Resid Cardiol. 2019;152:96-100.

24. Grant SW, Collins GS, Nashef SAM. Statistical Primer: developing and validating a risk prediction model. Eur J Cardiothorac Surg. 2018;54(2):203-8.

25. Magee MJ, Wright CD, McDonald D, Fernandez FG, Kozower BD. External validation of the Society of Thoracic Surgeons General Thoracic Surgery Database. Ann Thorac Surg. 2013;96(5):1734-9. 